\title{
Automatic Number Plate Recognition Systemusing Connected Component Analysis and Convolutional Neural Network
}

\author{
Sneh Kanwar Singh Sidhu, Raman Maini, Dhavleesh Rattan
}

\begin{abstract}
Technology is becoming constantly important for customers. Automatic number plate Recognition (ANPR) is a device which enables the identification of a number plate in real time. For an intelligent car service, ANPR helps to promote growth, customize the classic app and increase consumer and employee productivity. Within the specification, the principal function of ANPR lies of removing the characteristics from an illustration of a license plate. An application that enables customers to display automobile repairs through the license platform number only derived from a loaded picture is augmented by a smart car service. Technological progress is that, so it is thought that improvement is important in this region too, so the best choice for automotive services is a smart car company. This work proposed a methodology to detect the numbers from car license plate using convolutional neural network. In the preprocessing of photographs on license plates, the WLS and FFT filters were included. The images are then fed into the convolutional trainings neural network. On more plates and tests is reported during the testing. Therefore, the findings indicate that the proposed solution can be taken in less time from the license model to accurately identify the characters. The experimental result shows the significance of proposed research by achieving an accuracy of $98 \%$ for the localization and true recognition of license plates from the video frames.
\end{abstract}

Keywords: ANPR, FFT, WLS, CNN, plate Detection.

\section{INTRODUCTION}

Increasing population results in increasing vehicles and controlling these vehicles is a big problem. ANPR (Automatic Number Plate Recognition) system plays a vital role in effectively controlling vehicle related problems. It can find the vehicle and the person by tracking the number plate without direct human intervention. Also it is helpful in automatic ticketing of vehicles, tracking vehicles when they violate traffic rules like red light jump, access control in buildings and parking areas, toll collection, stolen car detection etc. with a huge saving of human time, effort and cost.

Manuscript received on August 28, 2021.

Revised Manuscript received on October 13, 2021.

Manuscript published on October 30, 2021.

* Correspondence Author

Sneh Kanwar Singh*, Department of Computer Science and Engineering, Punjabi University Patiala (Punjab), India. Email: snehkanwar26@gmail.com

Dr. Raman Maini, Professor Computer Science and Engineering, Punjabi University Patiala (Punjab), India. Email:Research_raman@yahoo.com

Dr. Dhavlessh Ratan, Assistant Professor, Computer Science and Engineering, Punjabi University Patiala (Punjab), India. Email: Dhavleesh@gmail.com

(C) The Authors. Published by Blue Eyes Intelligence Engineering and Sciences Publication (BEIESP). This is an open access article under the CC BY-NC-ND license (http://creativecommons.org/licenses/by-nc-nd/4.0/)
Aspect ratio (size of the number plate) is an important factor in number plates. Also the background color, font style, font size color of characters etc. should be following standard rules. Almost all developing and developed countries have same aspect ratio.

\section{A. ANPR (Automatic License Plate Recognition)}

It is a technology that recognizes the license plates of vehicles, using technologies like OCR (Optical Character Recognition) and other algorithms. It uses closed circuit television cameras or the road-rule enforcement cameras to capture the pictures and then algorithms are applied to the captured image to get the characters of the license plate. It is used by law enforcement agencies or the highway agencies like electronic tolls. ANPR system is used to find out the characters of the license plate and also the text on the licenseplate and may also capture the face of the driver. It also usesthe infrared cameras to capture the images even in bad lightning conditions like at night time. It is also able torecognize characters and text of the license plates of different regions or countries as there is plate variations from place toplace.

\section{B. Development of ANPR}

It was invented in Britain in 1976 at Police Scientific Development. Firstly in 1979 prototypes were built and EMIelectronics and CRS (Computer Recognition Systems) in Workingham (UK) were given the contracts to make the industrial systems. The system was implemented at A1 road at Dartford Tunnel and the first arrest was made for a stolen car in 1981. But ANPR was not much used in 1990s until the software was made easier and updated and the system was made cheaper. Also ANPR data was stored for future use and to solve cases like a 2005 murder case of Bartford, UK was solved using ANPR which helped to locate the murderers of Sharon Beshenivsky. With the development of science and technology, number of vehicles in the world are increasing, and management of vehicles is becoming very important. Theprevious manual management system needs a large number of operators to operate the system and also when the traffic ismore the manual system works very slow there should be anautomatic system that works on computer algorithms that do the work in less time and with more accuracy than humans. The modern approaches benefits from the high speed and lowerror rate of the computer, and has developed ANPR system to replace the traditional method [2].

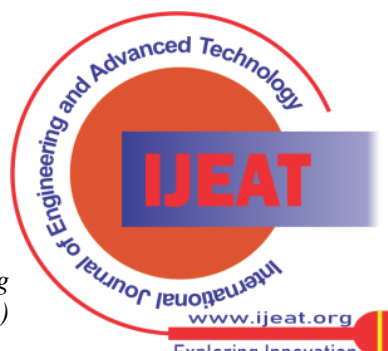




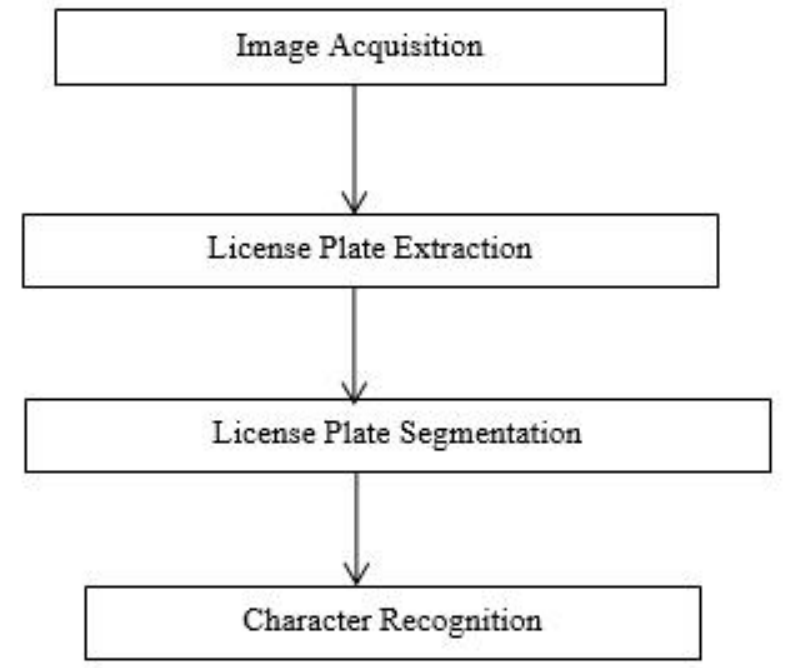

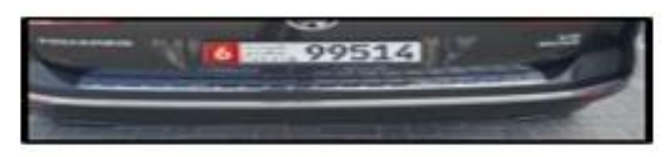
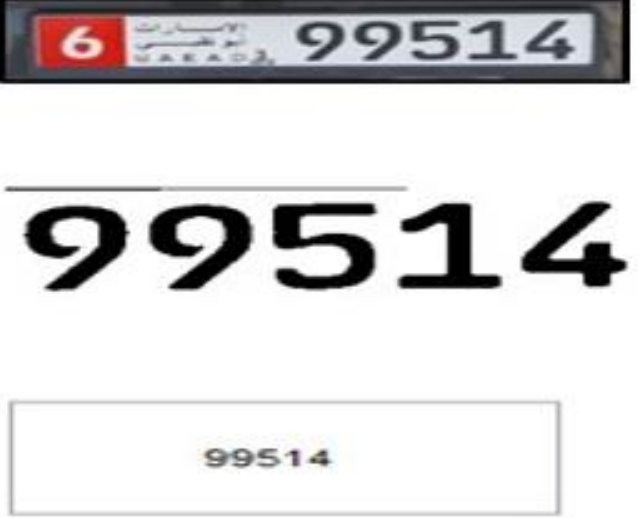

Fig. 1.The traditional four stages of a license plate recognition system

ANPR systems have many real time benefits, like it is used in parking areas, traffic management systems, toll plazas etc. ANPR systems [2] have four phases, as shown in Fig. 1. Phase one is image acquisition which consists of the input image captured by the camera. The phase two is to locate the number plate area from the photograph. The phase three is to segment the characters of the number plate. The fourth phase is the character recognition by template matching or the SVM classifier or CNN. The difficulty faced by ANPR systems is that the character recognition rate is not good in complex environments and skewed images [2]. The problem is to apply ANPR system on slow computing moving vehicles. So, the aim of this research is to build a good performing, accurate and lightweight ANPR system using CNN method that can perform the number plate recognition in complex environments and skewed angles as shown in Fig. 2.

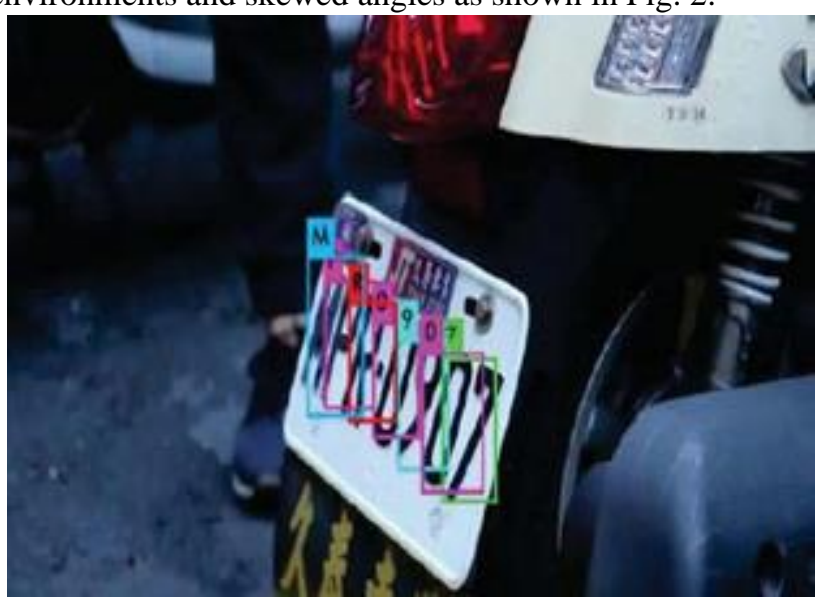

Fig. 2.License plate recognition example[1]

II.

LITERATURE REVIEW

Y. Y. Nguwi et. al (2015) [2] have preprocessed the image soas to detect the region of interest. The preprocessing step is to convert the image from RGB to grayscale first which results in eliminating the hue and saturation and retaining the luminance of the image. Then median filter is applied. RiazulIslam et. al (2015)[3]resized the image first to (400 X 600 ) size and then all processing steps will be done after converting the image into gray scale image. Contrast of the image is thus increased and the noise is removed. B.Pechiammal et al. (2017)[4] have converted the RGB image to gray scale after capturing the image and then applied Gabor filter to remove the noise.

Alan Koshy et al.(2019) [5] presents three techniques of pre-processing an image. First is thresholding an image which aims to binarize a gray scale image. Morphology is a technique by which image is examined by a structuring element. Blurring technique is used to remove high frequency contents in the image such as noise. Prathamesh Kulkarni et al.(2009) [6] have presented a method of plate localization by using a structured element of shape of inverted ' $\mathrm{L}$ ' because in Indian conditions number plates do not follow same rules.

Bhavin V Kakani et. al(2017) [7] used projection technique to clip the number plate. Vertical and horizontal band clipping are used. Riazul Islam etal. (2015)[3] have applied morphological operations to remove the irrelevant pixels from the image. Dilation and erosion are used to extract the desired license plate area. L.Angeline et. Al (2009)[8] makes use of normalization thatrefines the ROI into a block containing no extra pixels. This is necessary for template matching. Then the connected component technique is used after the image is complemented so that each white pixel can be labelled with 1,2,3 and so on and finally followed by inverting the image again. Monika A.Shejwal et.al(2017)[9] shows the segmentation method of text written in curved lines in a captured image. The bonding box method is used. It is a rectangle that is used to detect text lines by using connected component technique. Then $\mathrm{K}$ nearest neighbor technique is performed which uses Euclidean distance concept. Ali et al. (2017)[10] purpose was to increase the efficiency of the ANPR system by a good percentage by performing tests on several environments and situations.

Published By:

Blue Eyes Intelligence Engineering

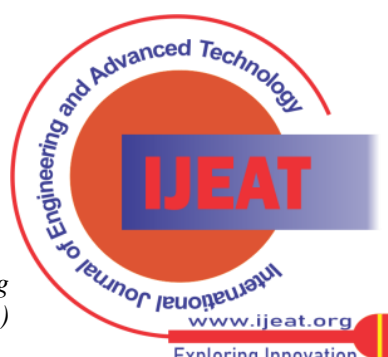


A few experiments are performed that will depict how good the system has improved on those different situations and circumstancesbased on various factors that have impact on the system.

The license plates used in these experiments are of different types like some have different font style, some have different background color, some have different texture etc. The license plates that do not follow the government standards are into experimentation and also the distance between the vehicle and the capturing camera is kept in consideration.

Therefore this system not only works for simple license but also with number plates having complex backgrounds and that do not follow the government standards. Regarding future scope the system should be improved by considering more factors like dim lights at night time. Various techniques to find the ROI should be taken into consideration.

Khare et al. (2019) [11] A reconstruction technique is presented by processing of the images of natural scenes, or from video frames. This technique uses laplacian and gradient features to get partial reconstruction for the characters to be segmented. This technique uses stroke width distance concept. The factors that create confusion and problems in processing like complex background, fog, mud, skewed images etc we provide angular information about the lartial reconstruction results that will help to get good results and will solve over and under segmentation. This technique uses stroke width distance method for the segmented letters to restore the partial reconstruction results. This technique is applied on a large dataset and also natural scene images and video frames are taken into consideration. Regarding future work this technique can be extended by making use of deep learning concept and also performing the experiment on different language scripts of different countries all over the world.

Chattopadhyay $\mathrm{T}$ et al. (2014) [12] proposed an image retrieval based method to detect the vehicle license plate taken using a smart phone camera to provide the vehicle management system of a Smart office premise. In the given technique a smart phone is used to acquire the images and extract features of the vehicle license plate. These features are compared with predefined set of same vehicle license plate images in the dataset. The character images are matched in an efficient way to make it a real time solution. The used technique detects the vehicle with approximate 93.75\% accuracy and is already working in our office premises. Quan. et al (2020) [13] presents a work in which pixel level disturbance is there in the image and these disturbances can be scattered all over the image or some portion of the image and to calculate the values of these pixel perturbration values appropriate algorithms are applied and images very near to the previous image is calculated that can confuse the neural networks. In this research experiment is done on the numberplates that contain physical perturbrations and work is done on those number plates. To simulate the spots on the number plate fixed size pixels are used to replace them and then the algorithm is performed on the number plates. This represents a difficult optimation problem and this genetic algorithm solves it with good success rates and accuracy.
III.

\section{PRESENT WORK}

The methodology implemented in the proposed work comprises of four main steps which are mentioned below:

- Image Acquisition

- License Plate Extraction

- License Plate Segmentation

- Character Recognition

\section{A. Image/Video Acquisition}

- Video acquisition - The digital camera of Sony Cyber-shot was used to capture the video for a private car park in the town of Patiala. In the early morning hours from 08:30am to 09:30 a.m. the government and trade offices showed a large number of traffic, so that all the car parks were held. The driver and the publicity firm that operates the parking lot have approved a variety of photos. Further processing in matlab software is performed after the video collection, and is a powerful method for video and image processing and analysis.

- $\quad$ Frame making- Frames are extracted from the video using the matlab command of the VideoReader. VideoReader generates object $\mathrm{v}$ from a file called filename to read the video data.

- Detection of rectangles - Because the numerical plate isoften written in rectangular block shape, the way to further processing is efficient. For this linked component analysis, the input color image is converted into a binary 0's and 1 ' image in order to use morphological border-box operation to detect the image rectangles. The height-width ratio is then utilized for maintaining rectangles and excluding variable-size rectangles. This move reduces the quality of knowledge to a much lower amount where only places of concern are left.

\section{B. WLS Filter}

This is a non-linear, smoothing and edge preserving filter. It stands for weighted least squares filter and was introduced in [41]. WLS filter can effectively capture details with the help of multi scale and edge preserving decomposition. WLS has been successfully applied to many applications, like image enhancement and image fusion. The weighted least squares filter can maintain balance between the blurring as well as sharpening as compared to rest of the edge-preserving filters such as bilateral filter. If there is an input image I, WLS filtercan get a smooth image $\mathrm{S}$ (coarsen version of I), and S should be maximally close to I. The filtered image Scan be obtainedby

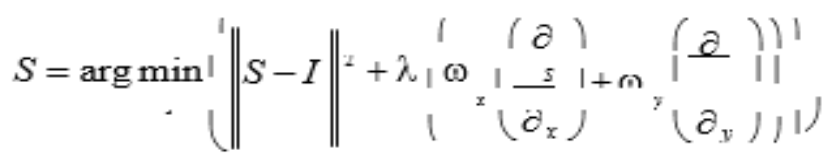

Where the data term $\|S-I\|^{2}$ enables the filtered image to be closer to the input image. And the regularization term is to achieve the smoothness by minimizing the partial derivatives of I. $\omega_{\mathrm{x}}$ and $\omega_{\mathrm{y}}$ are the horizontal and vertical smoothness weights. $\lambda$ is a regularization parameter which strikes a balance between the two terms.

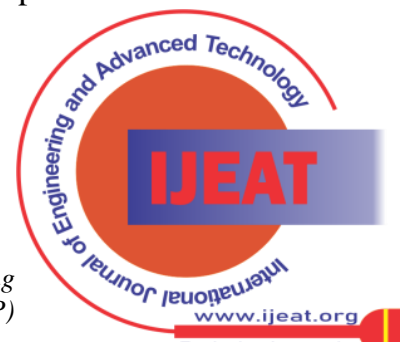




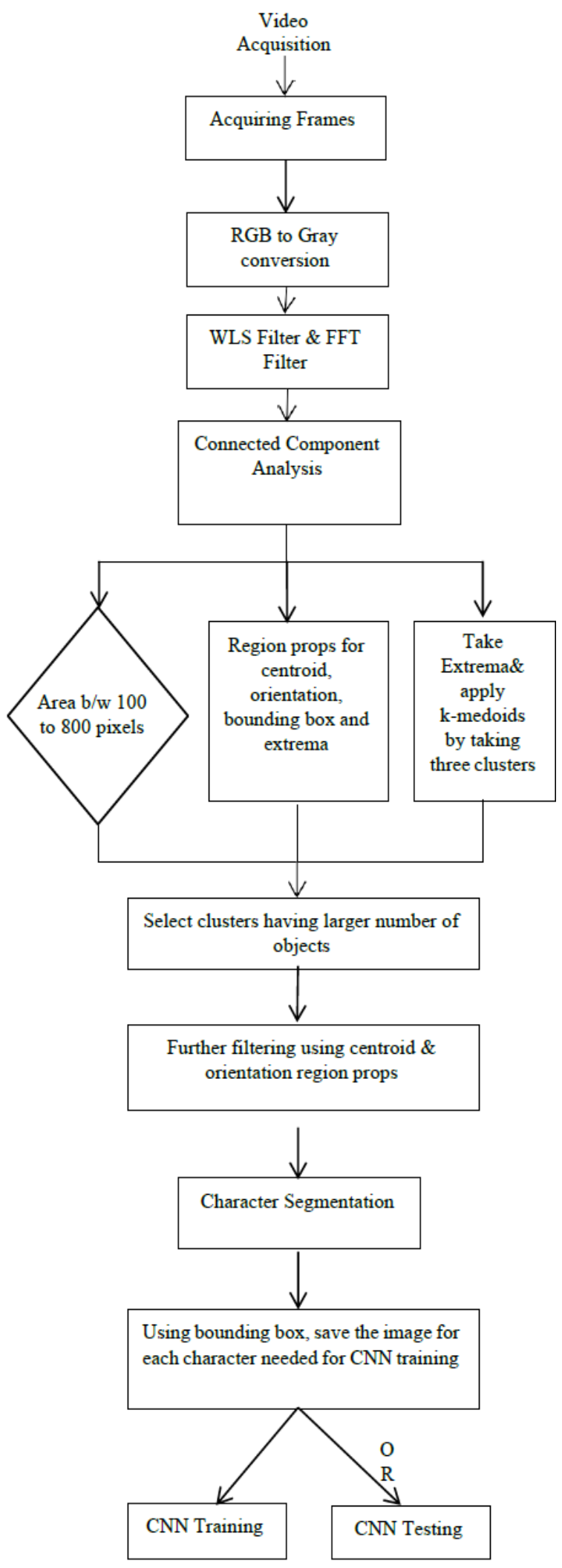

Fig. 3. Flowchart of proposed work

\section{FFT Filter}

FFT Filters allow accurate low and high-pass filtration, with a butterworth feature (smoothing and sharpening). The picture is transformed by Fast Fourier Transform into space frequencies, the filter is added, and the picture is inverted using FFT.

\section{Connected Component Analysis}

$\mathrm{L}$ = bwlabeln(BW) returns the matrix, L, which includes labels in the BW related components. The image of the inputBW is of the same size as BW. Integers are larger than or equal to 0 as elements of $\mathrm{L}$. The backdrop is the pixels numbered 0 . One object consists of the pixels labeled 1 ; the two pixels are a second object; and so forth. In 2 dimensions, the default connection is 8,3 dimensions 26 . In addition, the command regioprops is used to obtain various objects properties such as area, center pixel list, etc.

\section{E. Morphological Operations}

In this stage, the rim was connected to the defining picture to transform the image into 0 and 1 estimate. After this, collapse and expansion have been revised to delete funny pixels and preserve only the running objects. Such operations are performed as explained below. A morphology technique on a double image gives a pairing image in which the pixel has a non-zero value only if the check in the input picture is good in that region.

\section{F. Character Segmentation}

In the automatic plate detection and recognition (ALPDR) system, the process of character segmentation is unique, new and important because it depends on the functioning of all other processes. In comparison, when the segmentation process encounters errors, an inadequate division of the character into two parts or improper combination of two characters can occur, which in effect allows the identification process to fail. When the Automatic License Plate Detection and Recognition System (ALPDR) takes just a single row numerical plate into consideration, the segmentation mechanism can operate in defining horizontal borders between localized characters.

\section{G. Convolutional Neural Network}

A CNN model is generally composed of various layers such as convolution, pooling, normalization and fully connected layers. These layers are used sequentially to construct various architectures for various tasks. The convolution layers are employed to extract the local features from the input images[15].

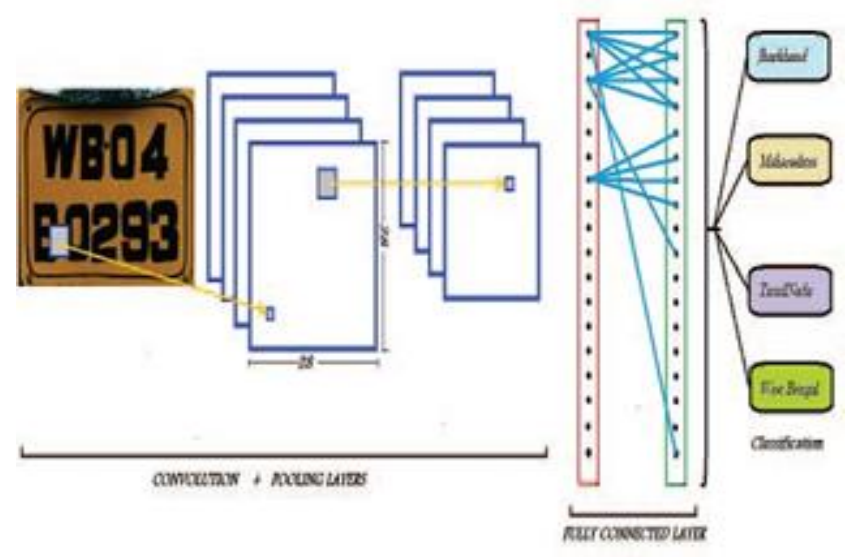

Fig. 4.CNN Architecture [16]

Published By:

Blue Eyes Intelligence Engineering

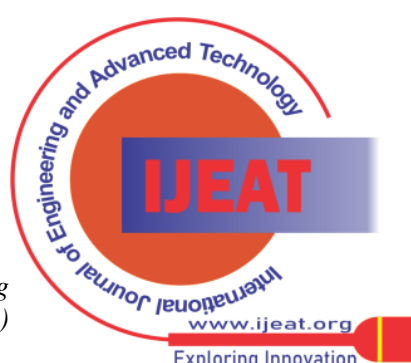


Four main operational layers are provided by $\mathrm{CNN}$ Convolution, ReLu (Non-linearity), Total pooling, and totally connected. The description of the suggested approach based on the CNN is shown in image 1. The Convolution method involves the featured layer creation by multiplying the filter values by the initial picture pixel values. Convolution layer inputs are smoothed such that the reactive design of the filters decreases to noise and shifts. The bundling method primarily decreases picture size or the color contrast between RGB channels. The CNN is consistent with broad several dynamic signal transmission features. The Rectified Linear nit (ReLU) is a popular feature that gains the quicker exercise tempo. The final layers of the network are completely linked, with neurons of previous layers in layers attached to each neuron[16].
This algorithm has been tried on various video frames captured from a private parking. For verifying our algorithm, it has been applied on different frames. Some of those frameshave been presented in the proposed work. The identification or license number for all motorized road vehicles in India is labelled. The identification address (number plate) for the caris given by their respective states' district-level RegionalTransport Office (RTO). In the front and behind the vehicle are the license plates. The license plates, by rule, all plaques must be issued Latin numerals in modern Hindu-Arabic. Additional requirements involve keeping the plate lit upthroughout the night and restricting font applications. Cars with exterior plates are not permitted to reach restricted areasin some states such as Sikkim. The term for India's overseas automobile registration is IND. Below is a step by stepdescription of the algorithm:

\section{EXPERIMENTAL SETUP}

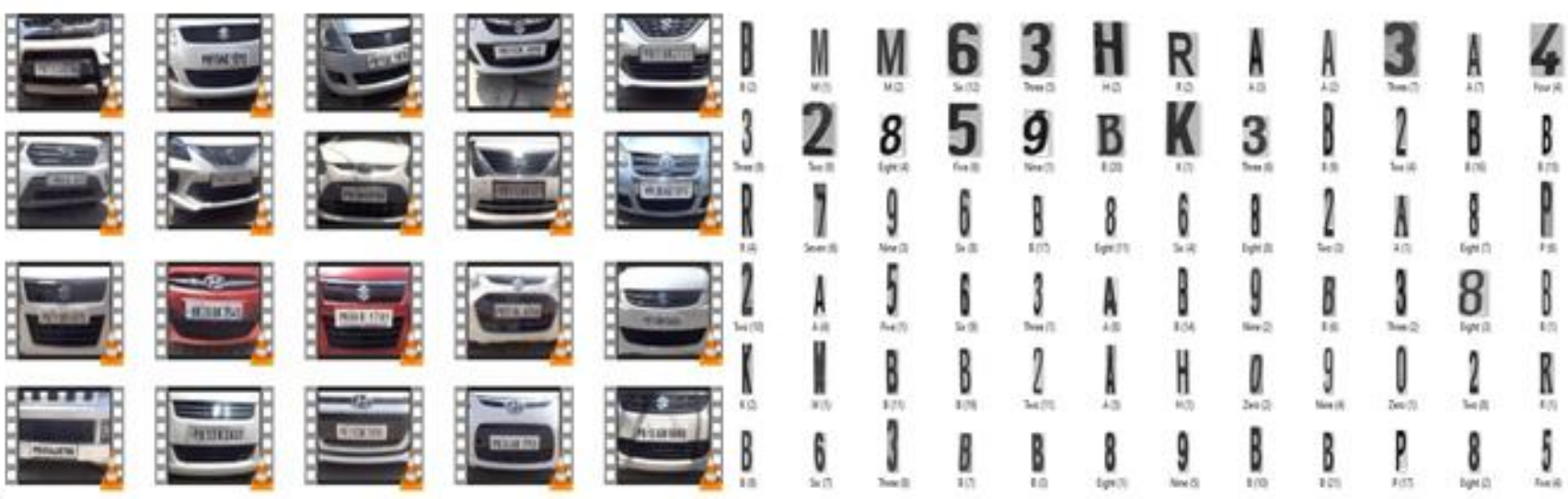

Fig. 7. Mp4 files collected for testing the proposed ALPR system. Fig. 8. A screenshot from characters folder that are
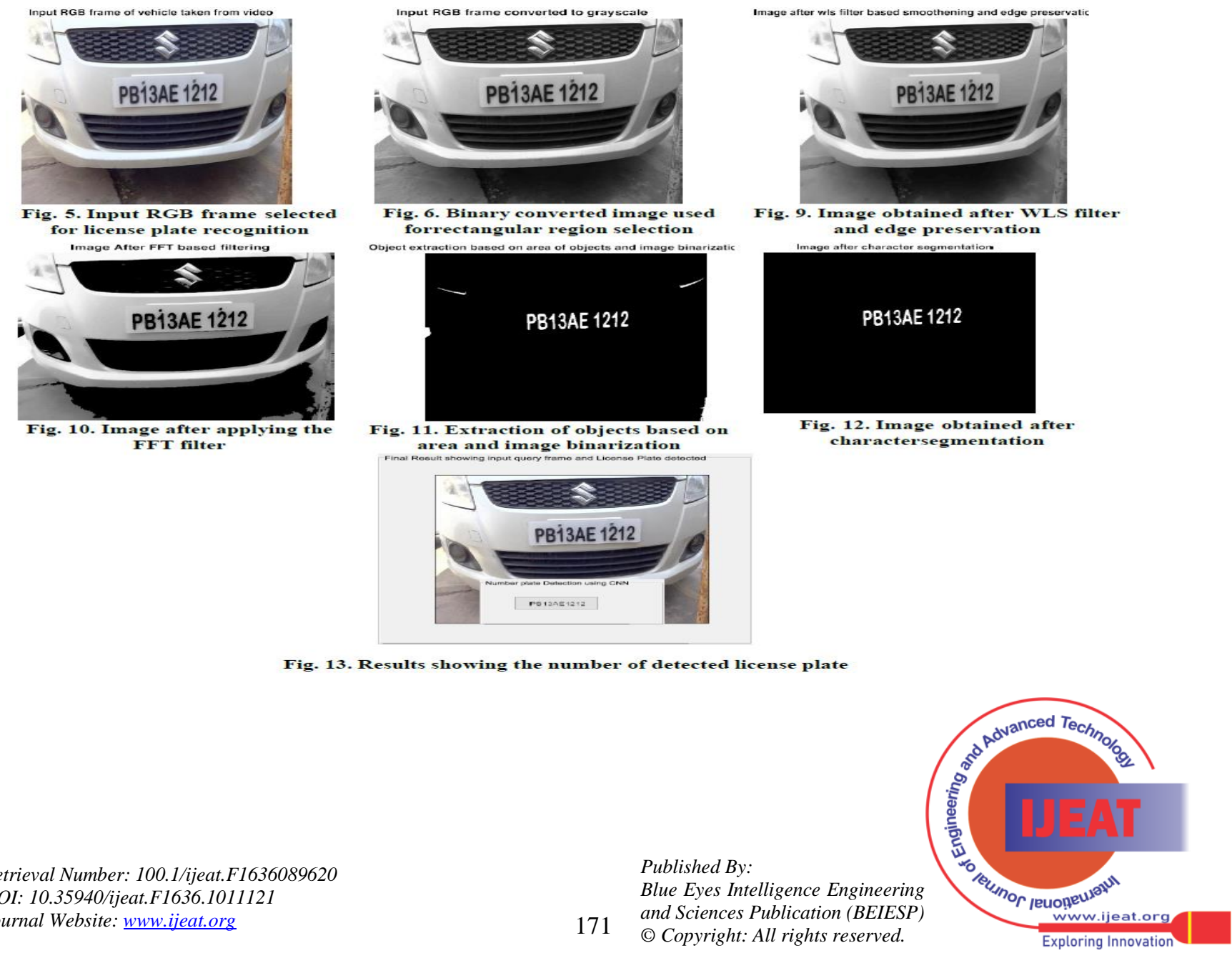


\section{Automatic Number Plate Recognition Systemusing Connected Component Analysis and Convolutional Neural Network}

\section{A. Classification Accuracy}

The consistency of the classification is to the degree that the extractor plate may correctly identify the specimens, and is resumed in the form of a complicated matrix for the research results. The number of characters identified after the license plaque detection device and the characters' realities on the basis of their localized sensitive and the specificity of the remainder of the scene, which is used as a backdrop, provide the efficacy of the approach provided. Hence detection accuracy (DA) specifying localization number plate charactersensitivity parameters will effectively reflect the precision of a proposed system for automatic license plate detector. Detection Accuracy (DA) is the number of (actually) locallydetected characters by a license plate detector system. Higheroutput requires greater precision of identification

$$
\text { DA_characters }=\frac{\text { \#Correctly det ected characters }}{\text { \#Total characaters in the number plate }} \times 100 \%
$$

The accuracy as per tested frames can be defined as:

$$
\text { DA frames }=\frac{\# \text { Frames in which license plate det ected }}{\# \text { Total number of frames tested }} \times 100 \%
$$

The below table shows the detection accuracy for both detected characters and detected plates from the video frames.

\begin{tabular}{|c|c|c|c|c|c|c|}
\hline $\begin{array}{c}\text { Number of Car } \\
\text { plate }\end{array}$ & $\begin{array}{l}\text { Number of } \\
\text { total characters }\end{array}$ & $\begin{array}{l}\text { Characters } \\
\text { detected } \\
\text { correctly }\end{array}$ & $\begin{array}{l}\text { DA of } \\
\text { characters }\end{array}$ & $\begin{array}{c}\text { Number of } \\
\text { total } \\
\text { frames } \\
\text { tested }\end{array}$ & $\begin{array}{c}\text { Number of } \\
\text { frames inwhich plate } \\
\text { is found/localize } d\end{array}$ & $\begin{array}{c}\text { DA of frames } \\
\text { that detectedthe } \\
\text { license plate }\end{array}$ \\
\hline PB13AE1212 & 10 & 10 & $100 \%$ & 40 & 40 & $100 \%$ \\
\hline PB11CU8786 & 10 & 10 & $100 \%$ & 40 & 40 & $100 \%$ \\
\hline PB11AL1978 & 10 & 10 & $100 \%$ & 40 & 40 & $100 \%$ \\
\hline PB11CN8418 & 10 & 10 & $100 \%$ & 40 & 40 & $100 \%$ \\
\hline PB11AV2727 & 10 & 9 & $90 \%$ & 40 & 39 & $98 \%$ \\
\hline PB65AL2835 & 10 & 10 & $100 \%$ & 40 & 40 & $100 \%$ \\
\hline PB11AV2727 & 10 & 9 & $90 \%$ & 40 & 39 & $98 \%$ \\
\hline PB19H0766 & 9 & 9 & $100 \%$ & 40 & 40 & $100 \%$ \\
\hline PB116V032 & 10 & 8 & $80 \%$ & 40 & 37 & $93 \%$ \\
\hline HR26AZ1513 & 10 & 10 & $100 \%$ & 40 & 40 & $100 \%$ \\
\hline PB11BP4575 & 10 & 9 & $90 \%$ & 40 & 38 & $95 \%$ \\
\hline HR26BR3545 & 10 & 9 & $90 \%$ & 40 & 38 & $95 \%$ \\
\hline PB34B1781 & 9 & 9 & $100 \%$ & 40 & 40 & $100 \%$ \\
\hline PB11BL6056 & 10 & 10 & $100 \%$ & 40 & 40 & $100 \%$ \\
\hline PB11BH0606 & 10 & 10 & $100 \%$ & 40 & 40 & $100 \%$ \\
\hline PB65AJ8789 & 10 & 10 & $100 \%$ & 40 & 40 & $100 \%$ \\
\hline PB13W2437 & 9 & 9 & $100 \%$ & 40 & 40 & $100 \%$ \\
\hline PB11CW2895 & 10 & 9 & $90 \%$ & 40 & 39 & $98 \%$ \\
\hline PB11AR77117 & 10 & 11 & $110 \%$ & 40 & 37 & $93 \%$ \\
\hline PB13AN9869 & 10 & 8 & $80 \%$ & 40 & 37 & $93 \%$ \\
\hline
\end{tabular}

Table 1: Table one showing different parameters for evaluating performance of the algorithm

Table 2: Approximate overall Accuracy of localizedplates in frames tested

\begin{tabular}{|c|c|}
\hline Method & Accuracy \\
\hline DA of characters & $96 \%$ \\
\hline Detection accuracy of frames in which licenseplates localized & $98 \%$ \\
\hline
\end{tabular}

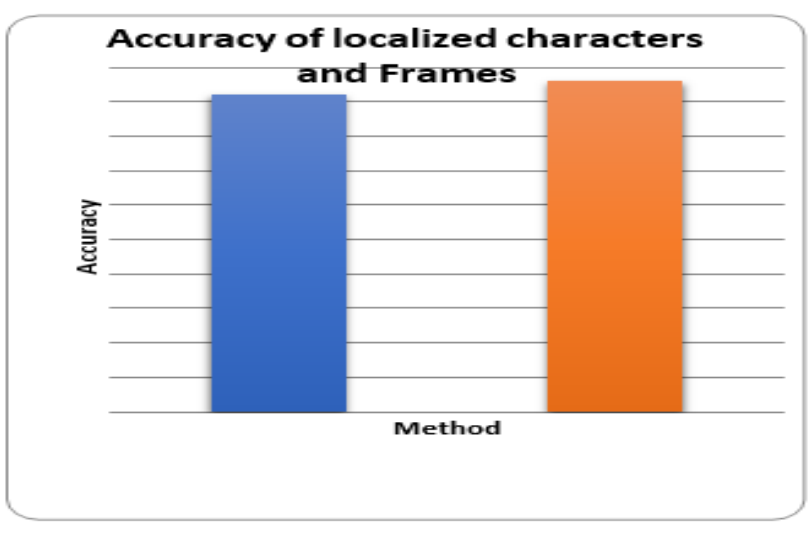

Fig. 14. Accuracy of localized characters and frames
Therefore, from the results it has been found that the proposed method is efficient in detecting the characters from the license plate accurately in less time. The highest accuracy found for this research work is $98 \%$ for the localized license plates from the video frames and accuracy for detecting the correct characters is $96 \%$.

\section{CONCLUSION}

The system suggested consists of two major steps, plate location / detection and identification of characters.

Published By:

Blue Eyes Intelligence Engineering and Sciences Publication (BEIESP)

(C) Copyright: All rights reserved.

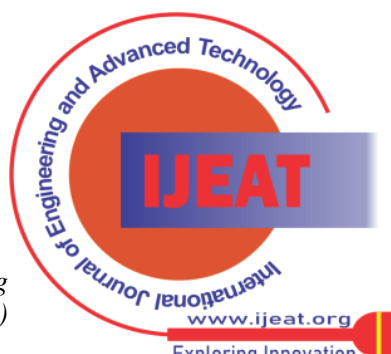


The Indian license plate has its unique features in order to modifyand improve any LPR method used for a good result. We used three different color spaces during the detection phase, whose components of intensity and chromaticity are concatenated. Binary picture is used to decrease the computer field of the image, where rectangular frames are first found and only those that have $\mathrm{h} / \mathrm{w}$ varies in the size of a license plate are recorded. The WLS and FFT filters were used to preprocess the images of license plates. After that the images are fed into the convolutional neural network for training. After the training the network is tested on more plates and results were recorded. The findings thus demonstrate that the approach suggested is effective in the correct identification of the characters in less time from the license plate. 98\% for localized license plates from the video frames were the highest accuracy found for the research work whereas the right characters are identified at $96 \%$ accurately.

\section{REFRENCES}

1. C. H. Lin and C. H. Wu, "A Lightweight, High-Performance MultiAngle License Plate Recognition Model," Int. Conf. Adv. Mechatron. Syst. ICAMechS, vol. 2019-Augus, pp. 235-240, 2019, doi: 10.1109/ICAMechS.2019.8861688.

2. Y. Y. Nguwi and W. J. Lim, "Number plate recognition in noisy image," Proc. - 2015 8th Int. Congr. Image Signal Process. CISP 2015, no. Cisp, pp. 476-480, 2016, doi: 10.1109/CISP.2015.7407927.

3. R. Islam, K. F. Sharif, and S. Biswas, "Automatic vehicle number plate recognition using structured elements," Proc. - 2015 IEEE Conf. Syst. Process Control. ICSPC 2015, no. December, pp. 44-48, 2016, doi: 10.1109/SPC.2015.7473557.

4. B. Pechiammal and J. A. Renjith, "An efficient approach for automatic license plate recognition system," ICONSTEM 2017 - Proc. 3rd IEEE Int. Conf. Sci. Technol. Eng. Manag., vol. 2018-Janua, pp. 121-129, 2017, doi: 10.1109/ICONSTEM.2017.8261267.

5. A. Koshy, "Extraction from Text Images," 2019 1st Int. Conf. Innov. Inf. Commun. Technol., pp. 1-4.

6. P. Kulkarni, A. Khatri, P. Banga, and K. Shah, "Automatic Number Plate Recognition (ANPR) system for Indian conditions," Proc. 19th Int. Conf. Radioelektronika 2009, RADIOELEKTRONIKA '09, pp. 111-114, 2009, doi: 10.1109/RADIOELEK.2009.5158763.

7. B. V. Kakani, Di. Gandhi, and S. Jani, "Improved OCR based automatic vehicle number plate recognition using features trained neural network," 8th Int. Conf. Comput. Commun. Netw. Technol. ICCCNT 2017, 2017, doi: 10.1109/ICCCNT.2017.8203916.

8. L. Angeline, K. T. . Teo, and F. Wong, "Smearing Algorithm for Vehicle Parking Management System," Proc. 2nd Semin. Eng. Inf. Technol., no. July, pp. 331-337, 2009.

9. M. A. Shejwal and S. D. Bharkad, "Segmentation and extraction of text from curved text lines using image processing approach," IEEE Int. Conf. Information, Commun. Instrum. Control. ICICIC 2017, vol. 2018-Janua, pp. 1-5, 2018, doi: 10.1109/ICOMICON.2017.8279138.

10. J. Ali et al., "Enhanced C ar N umber Plate R ecognition ( E C N P R) system by improving efficiency in pre- processing steps," vol. i, 2017.

11. V. Khare et al., "A novel character segmentation-reconstruction approach for license plate recognition," Expert Syst. Appl., vol. 131, pp. 219-239, 2019, doi: 10.1016/j.eswa.2019.04.030.

12. A. Visvanathan and T. Chattopadhyay, "Car number plate recognition for smart building management," Int. Conf. Digit. Signal Process. DSP, vol. 2014-Janua, no. August, pp. 73-77, 2014, doi: 10.1109/ICDSP.2014.6900802.

13. Y. Qian et al., "Spot evasion attacks: Adversarial examples for license plate recognition systems with convolutional neural networks," Comput. Secur., vol. 95, 2020, doi: 10.1016/j.cose.2020.101826.

14. Z. Farbman, R. Fattal, D. Lischinski, and R. Szeliski, "Edgepreserving decompositions for multi-scale tone and detail manipulation," SIGGRAPH'08 Int. Conf. Comput. Graph. Interact. Tech. ACM SIGGRAPH 2008 Pap. 2008, 2008, doi: 10.1145/1399504.1360666.

15. N. Omar, A. Sengur, and S. G. S. Al-Ali, "Cascaded deep learningbased efficient approach for license plate detection and recognition," Expert Syst. Appl., vol. 149, p. 113280, 2020, doi: 10.1016/j.eswa.2020.113280.

16. M. Mondal, P. Mondal, N. Saha, and P. Chattopadhyay, "Automatic number plate recognition using CNN based self synthesized feature learning," 2017 IEEE Calcutta Conf. CALCON 2017 - Proc., vol. 2018-Janua, pp. 378-381, 2018, doi:

10.1109/CALCON.2017.8280759.

\section{AUTHORS PROFILE}

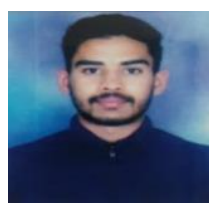

Sneh Kanwar Singh, Received his B.Tech Degree In Computer Engineering from Punjabi University, Patiala, India in May 2013 and Is pursuing Mtech in Computer Science and Engineering from Punjabi, University, Patiala, India. Area of Interest: Digital Image processing

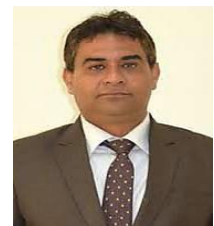

Dr. Raman Maini,, is a Well Qualified person and holds the Respective degrees of B.Tech, M.tech and PHD. He is designated as the HOD and Professor in Computer Science and Engineering Department in Punajbi University, Patiala, Punjab. He has Achieved a tremendous amount of Publications Ranging to 58. His area of interest is Digital Image processing, Networks, Software Engineering.

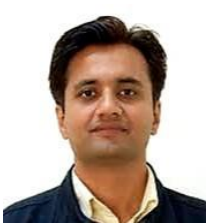

Dr. Dhavleesh Ratan, is a Well Qualified person and holds the Respective degrees of B.Tech, M.tech and PH.D. He is designated as the Assistant Professor in Computer Science and Engineering Department in Punajbi University, Patiala, Punjab. He has Achieved a tremendous amount of Publications Ranging to 16 . His area of interest is Network Security, Software Clone Detection, Software Testing, Digital Forensics, Web Crawlers.
Published By:

Blue Eyes Intelligence Engineering and Sciences Publication (BEIESP) (C) Copyright: All rights reserved.

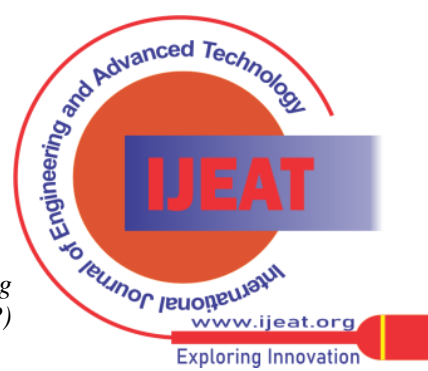

